Canadian Journal of Mathematics

doi:10.4153/CJM-2010-016-4

(C) Canadian Mathematical Society 2009

\title{
On the Spectrum of the Equivariant Cohomology Ring
}

\author{
Mark Goresky and Robert MacPherson
}

Abstract. If an algebraic torus $T$ acts on a complex projective algebraic variety $X$, then the affine scheme $\operatorname{Spec} H_{T}^{*}(X ; \mathbb{C})$ associated with the equivariant cohomology is often an arrangement of linear subspaces of the vector space $H_{2}^{T}(X ; \mathbb{C})$. In many situations the ordinary cohomology ring of $X$ can be described in terms of this arrangement.

\section{Introduction}

\subsection{Torus Actions and Equivariant Cohomology}

Suppose an algebraic torus $T$ acts on a complex projective algebraic variety $X$. If the cohomology $H^{*}(X ; \mathbb{C})$ is equivariantly formal (see $\$ 2$ ), then knowledge of the equivariant cohomology $H_{T}^{*}(X ; \mathbb{C})$ (as a module over $H_{T}^{*}(\mathrm{pt})$ ) is equivalent to knowledge of the ordinary cohomology groups, viz.

$$
\begin{aligned}
& H_{T}^{*}(X) \cong H^{*}(X) \otimes_{\mathbb{C}} H_{T}^{*}(\mathrm{pt}), \\
& H^{*}(X) \cong H_{T}^{*}(X) \otimes_{H_{T}^{*}(\mathrm{pt})} \text { C. }
\end{aligned}
$$

However the equivariant cohomology is often easier to understand as a consequence of the localization theorem [3]. For example, in [16] the equivariant cohomology ring $H_{T}^{*}(X ; \mathbb{C})$ of an equivariantly formal space $X$ was described in terms of the fixed points and the one-dimensional orbits, provided there are finitely many of each. In this paper we pursue the link between the equivariant cohomology and the orbit structure of $T$ by studying the affine scheme $\operatorname{Spc}_{T}^{*}(X)$ that is (abstractly) associated with the equivariant cohomology ring. Under suitable hypotheses, it turns out (Theorem 3.1) that the associated reduced algebraic variety $V$ is an "arrangement" of linear subspaces of the vector space $H_{2}^{T}(X)$. Many interesting arrangements arise this way.

The ring structure on the ordinary cohomology $H^{*}(X)$ can sometimes be recovered from the variety $V=\left(\operatorname{Spec} H_{T}^{*}(X)\right)_{\text {red }}$. This variety comes equipped with a finite linear mapping $\pi_{*}: V \rightarrow \mathrm{t}$ to the Lie algebra of $T$. For generic $t \in \mathrm{t}$ the fiber $\pi_{*}^{-1}(t)$ consists of finitely many points. If the mapping $\pi$ is flat and if $H^{*}(X)$ is generated by the part in degree $\leq 2$, we show (Theorem 4.1) that the filtration by degree on the coordinate ring $A_{t}=\mathbb{C}\left(\pi_{*}^{-1}(t)\right)$ induces an isomorphism of rings,

$$
\text { Gr } A_{t} \cong H^{*}(X) \text {. }
$$

Received by the editors March 31, 2007

Published electronically December 4, 2009.

The first author's research was partially supported by DARPA grant no. HR0011-04-1-0031.

AMS subject classification: 14L30, 54H15. 
This simple observation has interesting consequences in many different circumstances.

\subsection{Schubert Varieties}

For (possibly singular) Schubert varieties $X_{w}$, equation (1.1) becomes (Theorem 5.1) a theorem of Akyldiz-Carrell-Lieberman [1]:

$$
H^{*}\left(X_{w}\right) \cong \operatorname{Gr}\left(C\left(W_{\leq w} \cdot t\right),\right.
$$

where $t \in \mathrm{t}$ is regular, which was originally proved using the theory of holomorphic vector fields.

\subsection{Toric Varieties}

For non-singular toric varieties $X$, we show (Theorem 6.1) that the partially ordered set of cones in the fan $\Sigma$ defining $X$ coincides with the partially ordered set of flats (multi-intersections of the component subspaces) in the arrangement of linear spaces $V=\left(\operatorname{Spec} H_{T}^{*}(X)\right)_{\text {red }}$. In other words, it is possible to recover the fan $\Sigma$, in a simple way, from the equivariant cohomology $H_{T}^{*}(X)$. From this, we recover a theorem of Brion-Vergne [9]: the cohomology ring $H^{*}(X)$ is naturally isomorphic to the ring of piecewise polynomial functions on the fan $\Sigma$.

\subsection{Springer Fibers}

For Springer fibers $X_{a}$ in $G L(n, \mathbb{C})$ corresponding to a nilpotent element $a \in g l(n, \mathbb{C})$, we show (in Theorem 7.1) that equation (1.1) gives a theorem of J. Carrell [10] which identifies the cohomology ring $H^{*}\left(X_{a}\right)$ with the coordinate ring of the orbit of a certain point under the Weyl group. This result in turn was used by Carrell to give a short proof of the theorem of C. DeConcini and C. Procesi [14]: the coordinate ring of the scheme-theoretic intersection $\overline{C_{a}} \cap \mathrm{t}$ is isomorphic to the cohomology ring $H^{*}\left(X_{a}\right)$. (Here $\overline{C_{a}}$ is the closure of the conjugacy class of $a$.) Moreover, in Theorem 7.2 we show that the Springer representation of the Weyl group $W$ on $H^{*}(X)$ lifts naturally to a representation on the equivariant cohomology. (See also [16], where a similar result was proved in the case of affine Springer fibers.) The Springer representation is somewhat mysterious because $W$ does not act (in an algebraic way) on $X$, but rather it acts via deformations of $X[20,21]$. However, it turns out that the action of $W$ on the equivariant cohomology is easy to describe and that, in fact, the Weyl group can be naturally identified as the group

$$
W=\operatorname{Aut}_{\pi}(V)=\operatorname{Aut}_{\mathbb{C}[\mathrm{t}]} H_{T}^{*}(X),
$$

of automorphisms of the arrangement $V$ that preserve the projection $\pi$. The Springer representation on the ordinary cohomology may then be recovered from the isomorphism (1.2). 
On the Spectrum of the Equivariant Cohomology Ring

\subsection{Duality}

A nilpotent element $a \in \mathfrak{s l}(n, \mathbb{C})$ corresponds to a parabolic subgroup $P$ of $G=$ $S L(n, \mathbb{C})$ (or rather, to a class of associated parabolic subgroups). A Cartan subgroup $H \subset P$ acts on $G / P$, so we may consider the reduced affine scheme $V\left(\widehat{H}_{H}^{*}(G / P)\right)$, which is an arrangement of linear subspaces of $H_{2}^{H}(G / P)$. (Here $\widehat{H}^{*}$ denotes the part of the cohomology algebra that is generated by elements of degrees 0,2 .) In Theorem 8.1 we show that this arrangement is isomorphic to the "dual" of the arrangement $V\left(X_{a}\right) \subset H_{2}^{T}\left(X_{a}\right)$ that was considered in the preceding paragraph. We do not have a good explanation for this duality, and it would be interesting to know if there exist other examples of dual equivariant cohomology schemes.

Note added in proof: In the intervening years since this article was submitted for publication, a general setting for this duality has been developed by Braden, Licata, Phan, Proudfoot, and Webster [5].

\subsection{Related Articles and Acknowledgements}

Although many of the results and applications in this note can be extended to more general situations, the main object of this paper is to illustrate the use of $V=$ $\operatorname{Spec}\left(H_{T}^{*}(X)\right)$ as a unifying concept. It was implicitly considered in $[2,3]$, and explicitly in [6], [16, p. 27], [7, p. 83], and [8, Theorem 2]. We also wish to draw attention to the related articles $[5,11,19,22-26,32]$ and to the unrelated article [27].

Our interest in the scheme $V$ developed from joint work with Robert Kottwitz $[16,17]$ to whom we are grateful for many stimulating and rewarding discussions. We thank Tom Braden, Volker Puppe, Nick Proudfoot, Juliana Tymoczko, and an anonymous referee for their suggestions. The first author would also like to thank the Institute for Advanced Study for its support and hospitality.

\section{Definitions}

In this note, unless otherwise specified, all homology and cohomology groups will be taken with complex coefficients. The coordinate ring of an affine scheme $W$ over $\mathbb{C}$ is denoted $\mathbb{C}[W]$. Throughout this paper we fix an algebraic torus $T \cong\left(\mathbb{C}^{\times}\right)^{n}$ acting algebraically on a connected complex projective algebraic variety $X$ that is equivariantly embedded in some complex projective space. Let $E T \rightarrow B T$ be the universal principal $T$ bundle over the classifying space and let $H_{T}^{*}(X ; \mathbb{Z})=H^{*}\left(E T \times_{T} X ; \mathbb{Z}\right)$ and $H_{*}^{T}(X ; \mathbb{Z})=H_{*}\left(E T \times_{T} X ; \mathbb{Z}\right)$ denote the equivariant cohomology and equivariant homology (respectively) of $X$ with coefficients in the integers $\mathbb{Z}$.

Let $\chi^{*}(T)$ and $\chi_{*}(T)$ be the character and cocharacter groups, respectively, of $T$. The natural isomorphism

$$
\chi^{*}(T) \cong H^{2}(B T ; \mathbb{Z})=H_{T}^{2}(\mathrm{pt} ; \mathbb{Z})
$$

associates with each character $\lambda: T \rightarrow \mathbb{C}^{\times}$the first Chern class of the corresponding line bundle $L_{\lambda}$ on $B T$. We obtain an isomorphism

$$
\mathrm{t} \cong \chi_{*}(T) \otimes_{\mathbb{Z}} \mathbb{C} \cong H_{2}^{T}(\mathrm{pt} ; \mathbb{C})
$$


between the Lie algebra $t$ and the (second) equivariant homology of a point, as well as a degree-doubling isomorphism

$$
\mathbb{C}[\mathrm{t}] \cong H^{*}(B T ; \mathbb{C})=H_{T}^{*}(\mathrm{pt} ; \mathbb{C})
$$

between the graded ring of complex valued polynomials on $\mathrm{t}$ and the equivariant cohomology ring of a point. (This isomorphism is in fact defined over (0).)

The inclusion $\{\mathrm{pt}\} \hookrightarrow B T$ induces an augmentation

$$
\epsilon: \mathbb{C}[\mathrm{t}]=H^{*}(B T ; \mathbb{C}) \rightarrow H^{*}(\mathrm{pt} ; \mathbb{C})=\mathbb{C}
$$

whose kernel $\mathfrak{I} \subset \mathbb{C}[\mathrm{t}]$ is the augmentation ideal consisting of all polynomials whose constant term is 0 . A choice of basepoint in ET determines a mapping

$$
\iota: X \rightarrow X \times E T \rightarrow X \times_{T} E T
$$

with resulting homomorphism of $\mathbb{C}[\mathrm{t}]$-modules, $\iota^{*}: H_{T}^{*}(X ; \mathbb{C}) \rightarrow H^{*}(X ; \mathbb{C})$. The mapping $p: X \rightarrow\{\mathrm{pt}\}$ (of $X$ to a point) induces graded $(\mathbb{C}[\mathrm{t}]$-module homomorphisms

$$
p_{*}: H_{*}^{T}(X ; \mathbb{C}) \rightarrow H_{*}^{T}(\mathrm{pt} ; \mathbb{C}) \quad \text { and } \quad p^{*}: H_{T}^{*}(\mathrm{pt} ; \mathbb{C}) \rightarrow H_{T}^{*}(X ; \mathbb{C}) .
$$

Denote by $\pi_{*}: H_{2}^{T}(X ; \mathbb{C}) \rightarrow \mathrm{t}$ the restriction of $p_{*}$ to the degree 2 part.

Recall [16] that the action of $T$ on $X$ is equivariantly formal if the Leray-Serre spectral sequence

$$
E_{2}^{p q}=H^{p}\left(B T ; H^{q}(X ; \mathbb{C})\right) \Longrightarrow H_{T}^{p+q}(X)=H^{p+q}\left(X \times_{T} E T\right)
$$

for the fibration $h: X \times_{T} E T \rightarrow B T$ collapses, where $E T \rightarrow B T$ is the universal principal $T$-bundle. The following results are standard but we include their proofs for completeness.

Proposition 2.1 Suppose the action of $T$ on $X$ is equivariantly formal. Then the following statements hold.

(i) There is a (non canonical) isomorphism of graded $\mathbb{C}[\mathrm{t}]$ modules,

$$
H_{T}^{*}(X ; \mathbb{C}) \cong\left(\mathbb{C}[\mathrm{t}] \otimes_{\mathbb{C}} H^{*}(X ; \mathbb{C}) .\right.
$$

If the action of $T$ on $X$ is trivial, then this is an isomorphism of rings.

(ii) Regarding $\mathbb{C}$ as a $\mathbb{C}[t]$-module by extending scalars $\epsilon$ : $\mathbb{C}[\mathrm{t}] \rightarrow \mathbb{C}, \epsilon(f)=f(0)$, induces an isomorphism of $\mathbb{C}$-algebras,

$$
H^{*}(X ; \mathbb{C}) \cong H_{T}^{*}(X ; \mathbb{C}) \otimes_{\mathbb{C}[\mathrm{t}]} \mathbb{C}=H_{T}^{*}(X ; \mathbb{C}) / \mathfrak{I} H_{T}^{*}(X ; \mathbb{C}) .
$$

(iii) The following sequence of complex vector spaces is exact,

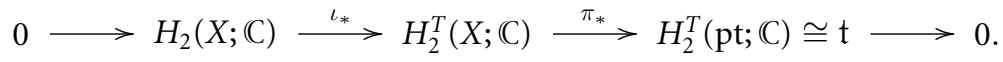

If $T$ acts with finitely many fixed points, then this sequence has a canonical splitting $\mu_{0}: \mathrm{t} \rightarrow H_{2}^{T}(X)$. 
(iv) The following sequence of $H_{T}^{*}(\mathrm{pt})$-modules is exact,

$$
0 \longrightarrow H_{T}^{*}(X) \longrightarrow H_{T}^{*}(F) \stackrel{\delta}{\longrightarrow} H_{T}^{*}\left(X_{1}, F\right),
$$

where $F=X^{T}$ is the fixed point set, and $X_{1} \subset X$ denotes the union of the 0 - and 1-dimensional orbits of $T$.

Proof The $E_{\infty}$ page of the spectral sequence (2.1) is the graded group associated with the filtration

$$
H^{m}(Y ; \mathbb{C})=F^{0} H^{m}(Y ; \mathbb{C}) \supset F^{1} H^{m}(Y ; \mathbb{C}) \supset \cdots \supset F^{m+1} H^{m}(Y ; \mathbb{C})=0,
$$

where $Y=X \times_{T} E T$ and where

$$
F^{k} H^{m}(Y ; \mathbb{C})=\operatorname{ker}\left[H^{m}(Y ; \mathbb{C}) \rightarrow H^{m}\left(h^{-1}\left(B T_{k-1} ; \mathbb{C}\right)\right)\right] .
$$

Here, $B T_{k}$ denotes the $k$-skeleton of $B T$ (with respect to some triangulation). Thus, for each $p, q$ there is a short exact sequence

$$
0 \longrightarrow F^{p+1} H^{p+q}(Y ; \mathbb{C}) \longrightarrow F^{p} H^{p+q}(Y ; \mathbb{C}) \stackrel{\alpha}{\longrightarrow} E_{\infty}^{p, q} \longrightarrow 0 .
$$

Moreover, the cup product takes

$$
F^{k} H^{m}(Y ; \mathbb{C}) \times F^{k^{\prime}} H^{m^{\prime}}(Y ; \mathbb{C}) \rightarrow F^{k+k^{\prime}} H^{m+m^{\prime}}(Y ; \mathbb{C})
$$

and induces a product on $E_{\infty}$ that is compatible with that on $E_{2}$ in the sense that the following diagram commutes:

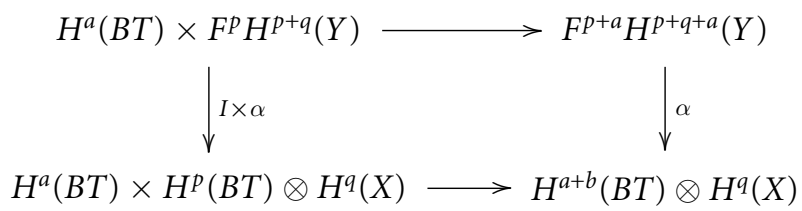

For this standard result see, for example, $[29, \S 9.4]$.

The classifying space $B T$ is simply connected and its cohomology in odd degrees vanishes, so $E_{2}^{p, q} \cong H^{p}(B T ; \mathbb{C}) \otimes H^{q}(X ; \mathbb{C})$ and $E_{2}^{p, q}=0$ if $p$ is odd.

Now assume the action is equivariantly formal so that $E_{\infty}^{p q}=E_{2}^{p q}$. A choice of splitting of each of the sequences (2.5) determines an isomorphism (2.2), which implies (i) and (ii). (If the action of $T$ on $X$ is trivial, then $Y \cong X \times B T$ is a product, and the Künneth theorem implies that (2.2) is a ring isomorphism.) The filtration

$$
H_{T}^{2}(X ; \mathbb{C})=F^{0} H^{2}(Y ; \mathbb{C}) \supset F^{1} H^{2}(Y ; \mathbb{C})=F^{2} H^{2}(Y ; \mathbb{C}) \supset F^{3} H^{2}(Y ; \mathbb{C})=0
$$


corresponds to a short exact sequence

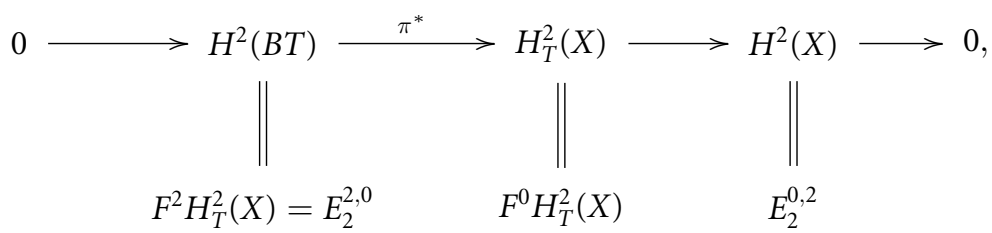

which implies (iii). If $T$ acts with finitely many fixed points then a canonical splitting of this sequence is defined in equation (3.2).

Part (iv) is the "topological part" of the localization theorem (see $[3,16,18]$ ).

Let $\widehat{H}^{*}(X)\left(\right.$ resp. $\left.\widehat{H}_{T}^{*}(X)=\widehat{H}^{*}(Y)\right)$ denote the subring of $H^{*}(X)\left(\right.$ resp. of $\left.H_{T}^{*}(X)\right)$ that is generated by the degree 0 and degree 2 part, $H^{0}(X) \oplus H^{2}(X)\left(\right.$ resp. $H_{T}^{0}(X) \oplus$ $\left.H_{T}^{2}(X)\right)$. Similarly let $\widehat{H}_{T}^{m}(X)=\widehat{H}_{T}^{*}(X) \cap H_{T}^{m}(X)$, and $F^{p} \widehat{H}^{a}(Y)=F^{p} H^{a}(Y) \cap \widehat{H}^{*}(Y)$ etc., where $Y=X \times_{T} B T$.

Proposition 2.2 (see also [23, p. 14]) Suppose $H^{*}(X)=\widehat{H}^{*}(X)$. Then the action of $T$ is equivariantly formal and $H_{T}^{*}(X)=\widehat{H}_{T}^{*}(X)$.

Proof Assume $H^{*}(X)=\widehat{H}^{*}(X)$. Then the cohomology of $X$ vanishes in odd degrees. Since the same is true for $H^{*}(B T)$, the spectral sequence (2.1) collapses and $E_{\infty}^{p, q} \cong H^{p}(B T) \otimes H^{q}(X)$. The filtration (2.5) is then given by

$$
F^{p} H^{m}(Y)=\bigoplus_{\substack{a+b=m \\ a \geq p}} H^{a}(B T) \otimes H^{b}(X)
$$

for any choice of splitting of (2.5).

The mapping $\phi: \mathbb{C}\left[H_{2}(Y)\right] \rightarrow \widehat{H}^{*}(Y)$ associates with any monomial on $H_{2}(Y)$ the corresponding product of cohomology classes in $H^{*}(Y)$. We claim this is surjective. Let $\mathbb{C}\left[H_{2}(Y)\right]^{m}$ denote the subspace of homogeneous polynomials of degree $m$. A choice of splitting of (2.4) gives an isomorphism

$$
\mathbb{C}\left[H_{2}(Y)\right]^{m} \cong \bigoplus_{a+b=m} \mathbb{C}[\mathrm{t}]^{a} \otimes \mathbb{C}\left[H_{2}(X)\right]^{b}
$$

Let

$$
F^{p}\left(\mathbb{C}\left[H_{2}(Y)\right]^{m}=\bigoplus_{\substack{a+b=m \\ a \geq p}} \mathbb{C}[\mathrm{t}]^{a} \otimes \mathbb{C}\left[H_{2}(X)\right]^{b}\right.
$$

We will show by induction on $p$ that the mapping $\phi$ takes $F^{P}\left(C\left[H_{2}(Y)\right]^{m}\right.$ surjectively 
On the Spectrum of the Equivariant Cohomology Ring

to $F^{p} H^{m}(Y)$. Using 2.6 , we have exact sequences

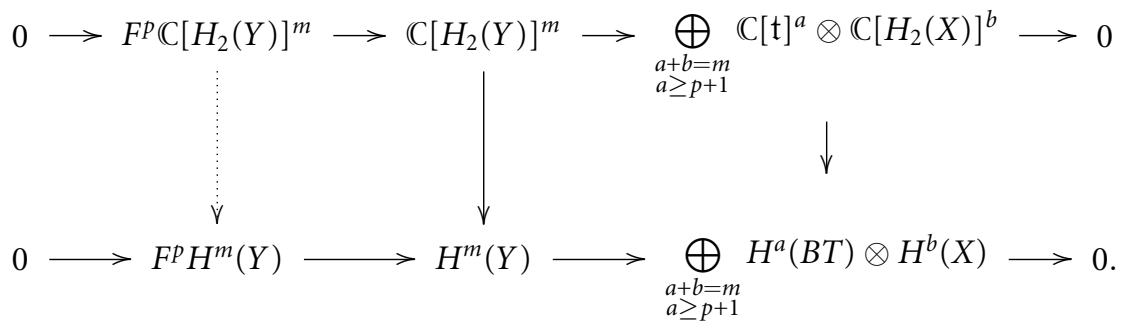

S

which shows that $\phi$ takes $F^{P}\left(C\left[H_{2}(Y)\right]^{m}\right.$ into $F^{p} H^{m}(Y)$. Let us assume by descending induction on $p$ that this mapping is surjective (with the case $p=m+1$ being trivial), and consider the exact sequences,

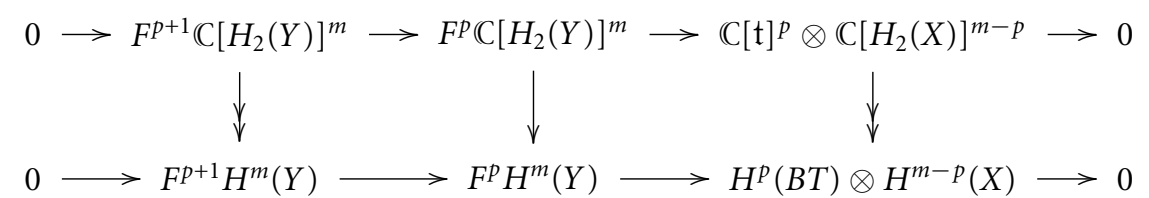

The right-hand vertical mapping is surjective by assumption. It follows that the middle vertical mapping is also surjective.

One might ask whether $\widehat{H}_{T}^{*}(X) \cong \mathbb{C}[\mathrm{t}] \otimes \widehat{H}^{*}(X)$ for equivariantly formal actions, but this statement is false for the Grassmannian $G_{2}\left(\mathbb{C}^{4}\right)$.

\section{Fixed Points and the Schemes $V$ and $W$}

As in Section 2, let $T$ be a complex algebraic torus acting on a complex algebraic variety $X$ that is $T$-equivariantly embedded in some projective space $\mathbb{P}^{N}$. Assume the fixed point set $F$ consists of finitely many connected components $F_{1}, F_{2}, \ldots, F_{k} \in X$. For each $i$, choose a point $x_{i} \in F_{i}$. Each inclusion $\left\{x_{i}\right\} \rightarrow X$ induces a splitting of (2.4),

$$
\mu_{i}: H_{2}^{T}\left(\left\{x_{i}\right\}\right) \cong \mathrm{t} \rightarrow H_{2}^{T}(X ; \mathbb{C})
$$

whose image is a linear subspace $\mathrm{t}_{i} \subset H_{2}^{T}\left(X ;(\mathbb{C})\right.$. The mapping $\mu_{i}$ is independent of the choice of point $x_{i} \in F_{i}$, however if $i \neq j$, then $\mathrm{t}_{i} \neq \mathrm{t}_{j}$ : the subspaces $\mathrm{t}_{i} \in H_{2}^{T}(X)$ are all distinct. (In fact, even the images of the $t_{i}$ in $H_{2}^{T}\left(\mathbb{P}^{N} ;(\mathbb{C})\right.$ are disjoint, as may be seen by a direct calculation.) The average of the mappings $\mu_{i}$ determines a canonical splitting,

$$
\mu_{0}=\frac{1}{k} \sum_{i=1}^{k} \mu_{i}: \mathrm{t} \rightarrow H_{2}^{T}(X)
$$


Denote by $V=\bigcup_{i=1}^{k} t_{i}$ the resulting arrangement of linear subspaces of the vector space $H_{2}^{T}\left(X ;(\mathbb{C})\right.$. (It is defined over $\left(\mathbb{O}_{2}\right.$.) We may consider $V$ to be a reduced affine scheme. Let

$$
\phi_{i}=\mu_{i}^{*}: \mathbb{C}\left[H_{2}^{T}(X)\right] \rightarrow \mathbb{C}\left[t_{i}\right] \cong H_{T}^{*}\left(\left\{x_{i}\right\}\right)
$$

be the resulting (surjective) map on functions. Then $I\left(t_{i}\right)=\operatorname{ker}\left(\phi_{i}\right)$ is the ideal of functions that vanish on $\mathrm{t}_{i}$ and $I(V)=\bigcap_{i=1}^{k} I\left(\mathrm{t}_{i}\right)$ is the (homogeneous) ideal of functions that vanish on $V$.

The identifications $H_{T}^{0}(X ; \mathbb{C}) \cong \mathbb{C}$ and $H_{T}^{2}(X ; \mathbb{C}) \cong \operatorname{Hom}\left(H_{2}^{T}(X ; \mathbb{C}), \mathbb{C}\right)$ determine a degree-doubling homomorphism of graded $(\mathbb{C}[\mathrm{t}]$-algebras,

$$
\mathbb{C}\left[H_{2}^{T}(X ; \mathbb{C})\right] \rightarrow H_{T}^{*}(X ; \mathbb{C})
$$

whose image is $\widehat{H}_{T}^{*}(X)$. Denote this surjection by $\theta: \mathbb{C}\left[H_{2}^{T}(X ; \mathbb{C})\right] \rightarrow \widehat{H}_{T}^{*}(X ; \mathbb{C})$. Similarly, let $\psi_{i}$ be the composition

$$
\mathbb{C}\left[H_{2}^{T}(X ; \mathbb{C})\right] \longrightarrow \widehat{H}_{T}^{*}(X ; \mathbb{C}) \longrightarrow \widehat{H}_{T}^{*}\left(F_{i} ;(\mathbb{C})\right.
$$

and let $R_{i}$ be the image of $\psi_{i}$. Let $W_{i}=\operatorname{Spec}\left(R_{i}\right)$. In summary we have the diagram

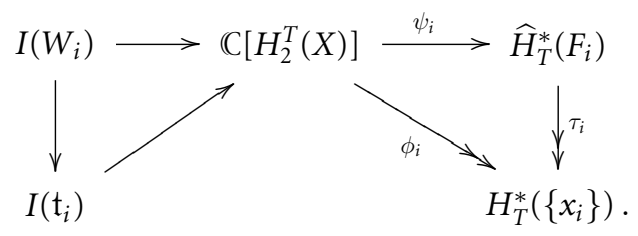

Let $W=\bigcup_{i=1}^{k} W_{i} \subset \mathbb{C}\left[H_{2}^{T}(X)\right]$ be the resulting affine scheme. It is defined by the ideal $I(W)$ in the following exact sequence:

$$
0 \longrightarrow I(W) \longrightarrow C\left[H_{2}^{T}(X ;(\mathbb{C})] \stackrel{\Sigma \psi_{i}}{\longrightarrow} \bigoplus_{i=1}^{k} R_{i}\right.
$$

The projection $\pi: X \rightarrow\{p t\}$ induces a mapping $\pi_{*}: W \rightarrow \mathrm{t}$ as the composition

$$
W \subset H_{2}^{T}(X) \rightarrow H_{2}^{T}(\{p t\}) \cong \mathrm{t}
$$

For $t \in \mathrm{t}$, denote the scheme-theoretic intersection $\pi_{*}^{-1}(t) \cap W$ by $W_{t}$. Let

$$
A_{t}=\mathbb{C}\left[W_{t}\right]=\mathbb{C}\left[H_{2}^{T}(X)\right] / I_{t}
$$

be the coordinate ring of $W_{t}$, where $I_{t}=I(W)+I\left(\pi_{*}^{-1}(t)\right)$. If $t=0$, then $I_{t}$ is a homogeneous ideal. 
Theorem 3.1 Suppose the algebraic torus $T$ acts on the projective variety $X$ with a fixed point set consisting of finitely many connected components $F_{1}, F_{2}, \ldots, F_{k} \subset X$. Let

$$
V=\bigcup_{i=1}^{k} \mathrm{t}_{i} \subset W=\bigcup_{i=1}^{k} W_{i} \subset H_{2}^{T}(X)
$$

be the resulting affine schemes. Then the following statements hold.

(i) The variety $V$ is the support of $W$, that is, $V=(W)_{\text {red. }}$.

(ii) If the fixed points are isolated then $W$ is reduced (so $V=W$ ).

(iii) If the action of $T$ on $X$ is equivariantly formal, then

(a) the homomorphism $\theta$ induces an identification

$$
W \cong \operatorname{Spec} \widehat{H}_{T}^{*}(X ; \mathbb{C})
$$

or equivalently, the following sequence is exact

$$
0 \longrightarrow I(W) \longrightarrow \mathbb{C}\left[H_{2}^{T}(X ; \mathbb{C})\right] \stackrel{\theta}{\longrightarrow} \widehat{H}_{T}^{*}(X ; \mathbb{C}) \longrightarrow 0,
$$

(b) the scheme $W$ is not contained in any proper subspace of $H_{2}^{T}(X)$, and

(c) there is a canonical isomorphism of rings,

$$
A_{0}=\mathbb{C}\left[W_{0}\right] \cong \widehat{H}_{T}^{*}(X) \otimes_{\mathbb{C}[\mathrm{t}]} \mathbb{C}
$$

(iv) If $H^{*}(X)=\widehat{H}^{*}(X)$, then the action of $T$ is equivariantly formal, the mapping $\pi_{*}: W \rightarrow \mathrm{t}$ is flat, and the isomorphism (3.5) becomes $A_{0} \cong H^{*}(X)$.

(v) In particular, if $H^{*}(X)=\widehat{H}^{*}(X)$ and the fixed points of $T$ are isolated, then $V=\operatorname{Spec} H_{T}^{*}(X)$ is reduced; it is an arrangement of linear spaces, and $H_{T}^{*}(X)$ is canonically identified with the algebra of functions on this arrangement.

Proof For part (i) it suffices to show that $\mathrm{t}_{i}=\left(W_{i}\right)_{\text {red }}$, that is, for any $f \in I\left(V_{i}\right)=$ $\operatorname{ker} \phi_{i}$ there exists $N>0$ such that $f^{N} \in I\left(W_{i}\right)=\operatorname{ker} \psi_{i}$. Given $f \in I\left(V_{i}\right)$, let $g=\psi_{i}(f)$. Since $T$ acts trivially on $F_{i}$, we have an isomorphism of rings

$$
\widehat{H}_{T}^{*}\left(F_{i}\right) \cong \widehat{H}^{*}\left(F_{i}\right) \otimes H_{T}^{*}(\{p t\}) .
$$

The element $g$ lies in the augmentation ideal, $\operatorname{ker}\left(\tau_{i}\right)$, that is, $g=\sum_{j} u_{j} \otimes v_{j}$ where $u_{j} \in \widehat{H}^{*}\left(F_{i}\right)$ has degree $\geq 2$ and $v_{j} \in H_{T}^{*}(\{p t\})$. Therefore $g^{\operatorname{dim}\left(F_{i}\right)+1}=0$, which is to say that $f^{\operatorname{dim}\left(F_{i}\right)+1} \in I\left(W_{i}\right)$, proving (i). Part(ii) follows immediately.

Now suppose the action of $T$ is equivariantly formal. By Proposition 2.1(iv), the restriction mapping $H_{T}^{*}(X ; \mathbb{C}) \rightarrow H_{T}^{*}(F ; \mathbb{C})$ is injective. It follows that $\widehat{H}_{T}^{*}(X ; \mathbb{C})$ becomes canonically identified with the image of $\psi$ in the following diagram,

$$
0 \longrightarrow I(W)=\bigcap_{i=1}^{k} I\left(W_{i}\right) \longrightarrow \mathbb{C}\left[H_{2}^{T}(X)\right] \stackrel{\psi}{\longrightarrow} \widehat{H}_{T}^{*}(F)=\bigoplus_{i=1}^{k} \widehat{H}_{T}^{*}\left(F_{i}\right)
$$


which proves (3.4). If $W$ were contained in some proper subspace $L \subset H_{2}^{T}(X)$, then there would exist a non zero linear function $\alpha: H_{2}^{T}(X) \rightarrow \mathbb{C}$ which vanishes on $W$ so the (homogeneous part of degree one in the) coordinate ring $\mathbb{C}[W]$ would fail to surject to $H_{T}^{2}(X)$. This proves (iii)(b).

Observe that $I\left(\pi_{*}^{-1}(0)\right)=\mathfrak{I} \mathbb{C}\left[H_{2}^{T}(X ; \mathbb{C})\right]$ (where $\mathfrak{I}$ denotes the augmentation ideal in $\mathbb{C}[t])$. So by equation (3.3) and (3.4),

$$
A_{0} \cong \widehat{H}_{T}^{*}(X) / \mathfrak{J} \widehat{H}_{T}^{*}(X) \cong \widehat{H}_{T}^{*}(X) \otimes_{\mathbb{C}[\mathrm{t}]}(\mathbb{C}
$$

which proves (iii)(c). Now assume that $H^{*}(X)=\widehat{H}^{*}(X)$. By Proposition 2.2 the action is equivariantly formal and $H_{T}^{*}(X)=\widehat{H}_{T}^{*}(X)$, so $W=\operatorname{Spec} H_{T}^{*}(X)$ which, by equation (2.2), is flat over $t$. Using (2.3), this gives a canonical isomorphism $A_{0} \cong$ $H^{*}(X)$.

Remark If $T$ acts with finitely many fixed points $x_{1}, \ldots, x_{k}$ and finitely many onedimensional orbits $E_{1}, \ldots, E_{r}$, then Theorem 7.2 of [16] identifies $\operatorname{Spec}\left(H_{T}^{*}(X ; \mathbb{C})\right)$ as the topological space obtained from the disjoint union $\coprod_{i=1}^{k} \mathrm{t}$ by identifying, for each one-dimensional orbit $E_{j}$, the subspace $t_{j}$ in the two copies of $t$ that correspond to the two "end points" of this orbit, where $t_{j}$ is the Lie algebra of the stabilizer of any point in the orbit $E_{j}$. (See also $[6,7]$.) Theorem 3.1 goes one step further and identifies this union as a particular affine scheme in $H_{2}^{T}(X)$.

\section{Specialization of the Coordinate Ring}

As in the preceding section, suppose the algebraic torus $T$ acts on the complex projective variety $X$ with isolated fixed points. Let

$$
W \longrightarrow H_{2}^{T}(X) \underset{\pi_{*}}{\longrightarrow} \mathrm{t}
$$

be the resulting scheme, supported on the arrangement $V$ of linear spaces, and its projection to $t$. Fix $t \in \mathrm{t}$ and let $W_{t}=W \cap \pi_{*}^{-1}(t)$ be the scheme-theoretic intersection with the coordinate ring $A_{t}=\mathbb{C}\left[W_{t}\right]$.

Let $A_{t}^{0} \subset A_{t}^{\leq 1} \subset \cdots$ be the filtration of $A_{t}$ by degree, that is, $A_{t}^{\leq k}$ consists of the restrictions to $V_{t}$ of the polynomials of degree $\leq k$. Let $\mu: H_{2}^{T}(X ; \mathbb{C}) \rightarrow H_{2}(X ; \mathbb{C})$ be a choice of splitting of (2.4). (A canonical such splitting $\mu_{0}$ is given in $₫ 3$.)

Theorem 4.1 (see also [11] and [25, p. 131]) Suppose the map (4.1) is flat. Then the splitting $\mu$ induces an isomorphism,

$$
\operatorname{Gr}\left(A_{t}\right) \cong A_{0}
$$

and different splittings give rise to the same isomorphism. If $\widehat{H}^{*}(X)=H^{*}(X)$, then Corollary 3.1 gives an isomorphism of rings,

$$
\operatorname{Gr}\left(A_{t}\right) \cong H^{*}(X)
$$


On the Spectrum of the Equivariant Cohomology Ring

Proof (This result is probably standard but we were not able to find a simple reference.) We may assume $t \neq 0$. Because the mapping $\pi_{*}$ is flat, by restricting to the line spanned by $t \in \mathrm{t}$ we may reduce to the case that $\mathrm{t}$ is one-dimensional. Choose an isomorphism of complex vector spaces $t \cong \mathbb{C}$. The splitting $\mu$ determines a splitting

$$
H_{2}^{T}(X ; \mathbb{C}) \cong H_{2}(X ; \mathbb{C}) \oplus \mathbb{C} .
$$

By further choosing a basis of $H_{2}(X ; \mathbb{C})$, we may consider each polynomial function $f=f(x, b) \in \mathbb{C}\left[H_{2}^{T}(X ; \mathbb{C})\right]$ to be a function of variables $x=\left(x_{1}, x_{2}, \ldots, x_{r}\right) \in$ $H_{2}(X ; \mathbb{C})$ and $b \in \mathbb{C}$, and we will sometimes write $\mathbb{C}\left[H_{2}^{T}(X ; \mathbb{C})\right]=\mathbb{C}[x, b]$. Specializing $b=0$ or $b=t$ gives two ring homomorphism $\mathbb{C}\left[H_{2}^{T}(X ; \mathbb{C})\right] \rightarrow \mathbb{C}\left[H_{2}(X ;(\mathbb{C})]\right.$ and we denote the image of $I(W)$ by $I_{0}, I_{t}$, respectively, in $\mathbb{C}\left[H_{2}(X ; \mathbb{C})\right]=\mathbb{C}[x]$. Then $A_{t}=\mathbb{C}[x] / I_{t}$ and $A_{0}=\mathbb{C}[x] / I_{0}$. The ideal $I_{0}$ is homogeneous and graded by degree, $I_{0}=\bigoplus_{j} I_{0}^{j}$, while the ideal $I_{t}$ is filtered by degree, $I_{t}^{0} \subset I_{t}^{\leq 1} \subset \cdots$. Therefore for each $k$,

$$
\begin{aligned}
& I_{0}^{k}=\left\{p(x) \in \mathbb{C}[x]: \operatorname{deg}(p)=k \text { and } \exists q(x, b) \in \mathbb{C}[x, b] \text { with } p+b q \in I(W)^{k}\right\} \\
& I_{t}^{\leq k}=\{p(x) \in \mathbb{C}[x]: \operatorname{deg}(p) \leq k \text { and } \exists q(x, b) \in \mathbb{C}[x, b] \\
&\text { with } \left.p+(b-t) q \in I(W)^{\leq k}\right\} .
\end{aligned}
$$

Observe that if $p \in I_{t}^{\leq k}$, then its homogeneous part $p_{k}$ of degree $k$ satisfies $p_{k} \in I_{0}^{k}$. (For there exists $q \in \mathbb{C}[x, b]$ of degree $\leq k-1$ such that $p+(b-t) q \in I(W)$. But $I(W)$ is homogeneous, so the degree $k$ homogeneous part $p_{k}+b q_{k-1}$ of this polynomial is also in $I(W)$, hence $p_{k} \in I_{0}^{k}$.) From this it follows that

$$
I_{0}^{k}=\left\{p \in \mathbb{C}[x]^{k}: \exists q \in \mathbb{C}[x]^{\leq k-1} \text { with } p+q \in I_{t}^{\leq k}\right\} .
$$

We now construct the required isomorphism

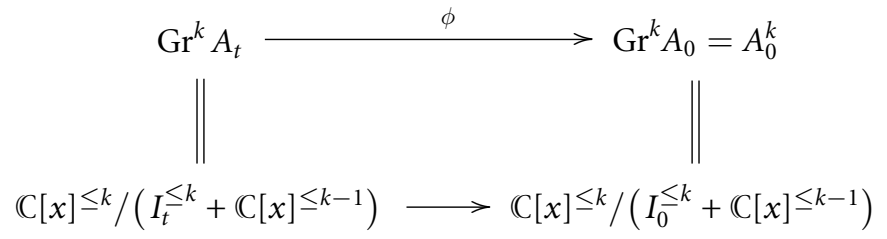

as follows. The identity mapping $\mathbb{C}[x] \leq k \rightarrow \mathbb{C}[x]^{\leq k}$ takes $I_{t}^{\leq k}$ to $I_{0}^{\leq k}+\mathbb{C}[x] \leq k-1$, for if $p \in I_{t}^{\leq k}$, then $p=p_{k}+p_{\leq k-1}$ and (by the above observation), $p_{k} \in I_{0}^{\leq k}$. This gives a well-defined surjective homomorphism

$$
\mathbb{C}[x]^{\leq k} / I_{t}^{\leq k} \rightarrow \mathbb{C}[x]^{\leq k} /\left(I_{0}^{\leq k}+\mathbb{C}[x]^{\leq k-1}\right)
$$

and hence also a surjective homomorphism $\phi$ above. To show that $\phi$ is injective, suppose $p \in \mathbb{C}[x]^{\leq k}$ is in the kernel of $\phi$. Then either $\operatorname{deg}(p)<k$ (in which case $p=0$ in $\left.\mathrm{Gr}^{k}\left(A_{t}\right)\right)$ or else $p_{k} \in I_{0}^{k}$. From equation (4.2) it follows that there exists $q \in \mathbb{C}[x]^{\leq k-1}$ such that $p_{k}+q \in I_{t}^{\leq k}$. Thus, $p+q=\left(p_{k}+q\right)+p \leq k-1 \in I_{t}^{\leq k}+\mathbb{C}[x] \leq k-1$ as needed. 
Theorem 4.1 is most useful when $T$ acts with finitely many fixed points and $t \in \mathrm{t}$ is generic, for in this case $W_{t}=V_{t}$ is reduced and consists of $k$ isolated points in the vector space $H_{2}^{T}(X)$. In the next few sections we will see what this says in the case of Springer fibers, Schubert varieties, and toric varieties, recovering results of $[1,10]$.

\section{Schubert Varieties}

Let $G$ be a complex semi-simple algebraic group. Let $B$ be a Borel subgroup, and set $X=G / B$. The Schubert variety $X_{w}$ is the closure of the Bruhat cell $B w B / B \subset X$ corresponding to an element $w \in W$ of the Weyl group. It is the disjoint union of Bruhat cells $X_{w}=\coprod_{\tau} B \tau B / B$, over those $\tau \in W_{\leq w}=\{\tau \in W: \tau \leq w\}$ (with respect to the strong Bruhat order on $W)$. Hence $X_{w}$ is equivariantly formal with respect to any torus action, and $H^{*}(X) \rightarrow H^{*}\left(X_{w}\right)$ is surjective, so $\widehat{H}^{*}\left(X_{w}\right)=H^{*}\left(X_{w}\right)$.

The Cartan subgroup $H \subset B$ acts on $X_{w}$ with a single fixed point in each of the Bruhat cells contained in $X_{w}$. Let

$$
V \subset H_{2}^{H}\left(X_{w}\right) \stackrel{\pi_{*}}{\longrightarrow} H_{2}^{H}(\mathrm{pt})=\mathfrak{h}
$$

be the resulting union of linear subspaces. Then, according to Theorem 4.1

$$
V \cong \operatorname{Spec} H_{H}^{*}\left(X_{w}\right)
$$

is a reduced affine scheme, and for any $t \in \mathfrak{h}=\operatorname{lie}(H)$ there is a canonical isomorphism

$$
\mathrm{Gr}\left(A_{t}\right) \cong H^{*}\left(X_{w}\right)
$$

where $A_{t}=A\left(V_{t}\right)$ is the coordinate ring of the scheme theoretic intersection $V \cap$ $\pi_{*}^{-1}(t)$. This gives a theorem of [1].

Theorem 5.1 Let $t \in \mathfrak{h}$ be a generic point. Then

$$
V_{t}=\bigcup_{\tau \in W_{\leq w}} w \cdot t \subset H_{2}\left(X_{w}\right)
$$

is a reduced affine scheme consisting of finitely many points. The filtration by degree on its coordinate ring $A_{t}=\mathbb{C}\left[H_{2}\left(X_{w}\right)\right] / I\left(V_{t}\right)$ induces an isomorphism of rings

$$
\mathrm{Gr} A_{t} \cong H^{*}\left(X_{w}\right)
$$

Proof The action of $H$ on $X_{w}$ is equivariantly formal, it has isolated fixed points, and $\widehat{H}^{*}\left(X_{w}\right)=H^{*}\left(X_{w}\right)$. So by Theorem 3.1 the corresponding scheme

$$
V=\operatorname{Spec} H_{H}^{*}\left(X_{w}\right) \subset H_{H}^{2}\left(X_{w}\right)
$$

is reduced and the projection $\pi: V \rightarrow \mathfrak{h}$ is flat. Therefore Theorem 7.1 will follow immediately from Theorem 4.1, provided we can establish the identification of $V_{t}$ with the collection of points 5.1. 
In the commutative diagram

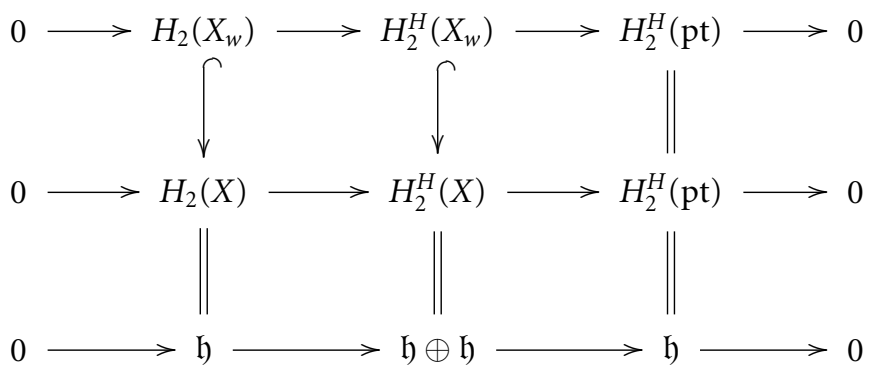

the splitting $\mu_{\tau}: H_{2}^{H}(\mathrm{pt}) \rightarrow H_{2}^{H}(X)$ corresponding to a fixed point $\tau x_{0} \in X(\tau \in W)$ is given by

$$
(\tau t, t) \leftarrow t
$$

(as the result of a calculation that we omit). Therefore, as a subset of $\mathfrak{h} \oplus \mathfrak{h}, V=$ $\left\{(\tau a, a): a \in \mathfrak{h}\right.$ and $\left.\tau \in W_{\leq w}\right\}$. In fact, $V$ is contained in the smaller vector space $H_{2}^{H}\left(X_{w}\right)$ and $V_{t} \subset H_{2}\left(X_{w}\right)$, but its coordinate ring is independent of the embedding.

\section{Toric Varieties}

As in Section 2, let $T \cong\left(\mathbb{C}^{\times}\right)^{n}$ be a complex algebraic torus with Lie algebra t. Let

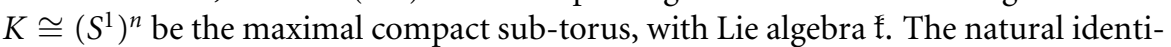
fication of character groups

$$
\chi^{*}(K) \cong \chi^{*}(T) \cong H_{T}^{2}(\mathrm{pt} ; \mathbb{Z})
$$

determines canonical isomorphisms

$$
\mathfrak{f}=\chi_{*}(K) \otimes_{\mathbb{Z}} \mathbb{R} \cong H_{2}^{T}(\mathrm{pt} ; \mathbb{R}) .
$$

As in Section 2, if $T$ acts on a topological space $X$, then the mapping $\pi: X \rightarrow \mathrm{pt}$ induces a homomorphism

$$
H_{2}^{T}(X ; \mathbb{R}) \rightarrow H_{2}^{T}(\mathrm{pt} ; \mathbb{R}) \cong \mathfrak{f},
$$

which we denote by $\pi_{*}$.

Suppose $X$ is a normal projective torus embedding or "toric variety" corresponding to a complex algebraic torus $T$ and a (finite) rational polyhedral cone decomposition $\Sigma$ of $\chi_{*}(T) \otimes_{\mathbb{Z}} \mathbb{R} \cong \mathfrak{f}$, as in $[4,15]$.

There is a simple relationship between the cone decomposition $\Sigma$ and the affine variety $V=\operatorname{Spec}\left(H_{T}^{*}(X ; \mathbb{C})\right)$. Using the canonical identification $\mathfrak{f} \cong \chi_{*}(T) \otimes_{\mathbb{Z}}$ $\mathbb{R}$, we obtain a rational polyhedral cone decomposition, which we also denote by $\Sigma$, of $⿱ ㇒$. Define a section $\Phi: \mathfrak{f} \rightarrow H_{2}^{T}(X ; \mathbb{R})$ which is linear on each cone as follows. The top dimensional (open) cones $C_{1}, \ldots, C_{k}$ correspond to the fixed points 
$F_{1}, \ldots, F_{k}$ of the torus action. For each of these $(1 \leq j \leq k)$, there is a splitting (3.1) $\mu_{j}: \mathfrak{l} \rightarrow H_{2}^{T}(X ; \mathbb{R})$ of equation (2.4), whose image we denote by $\mathfrak{f}_{j}$. Let

$$
V_{\mathbb{R}}=\bigcup_{i=1}^{k} \mathfrak{f}_{j} \subset H_{2}^{K}(X ; \mathbb{R})=H_{2}^{T}(X ; \mathbb{R})
$$

denote the resulting real arrangement of linear subspaces. It is stratified into "flats" by the multi-intersections of the subspaces $\mathfrak{f}_{i}$.

Let $L_{1}, L_{2}, \ldots, L_{m}$ denote the one-dimensional cones in $\Sigma$, and, for each $i$, choose a non-zero vector $v_{i} \in L_{i}$. Define $\Phi(x)=\mu_{j}(x)$ for all $x \in \bar{C}_{j}$ (the closure of the cone $C_{j}$ ).

Theorem 6.1 The mapping $\Phi: \mathfrak{f} \rightarrow H_{2}^{T}(X ; \mathbb{R})$ is continuous and linear on each cone. For each maximal cone $C_{i}$ the linear span of $\Phi\left(C_{i}\right)$ is the linear space $\mathfrak{f}_{i}$. If $X$ is rationally nonsingular, then the vectors $\Phi\left(v_{1}\right), \ldots, \Phi\left(v_{m}\right)$ form a basis of $H_{2}^{T}(X ; \mathbb{R})$, and the partially ordered set of flats in the arrangement $V_{\mathbb{R}}$ coincides with the partially ordered set of cones in $\Sigma$.

Proof First we verify the continuity of $\Phi$. We will show, for any codimension one cone $\tau$ in $\Sigma$, that

$$
\phi_{a}\left|\tau=\phi_{b}\right| \tau,
$$

where $C_{a}$ and $C_{b}$ are the two open cones such that $\bar{\tau}=\bar{C}_{a} \cap \bar{C}_{b}$. Such a cone $\tau$ corresponds to a one-dimensional orbit $E_{\tau} \subset X$ whose endpoints $F_{a}, F_{b} \in F$ are the fixed points corresponding to the open cones $C_{a}$ and $C_{b}$. Let $\mathfrak{f}_{\tau} \subset \mathfrak{f}$ be the Lie algebra of the stabilizer in $K$ of any point in $E_{\tau}$. Define the mapping

$$
\psi_{\tau}: \mathfrak{f}_{\tau} \rightarrow H_{2}(F ; \mathbb{R}) \cong \bigoplus_{i=1}^{k} \mathfrak{f}
$$

by $\psi_{\tau}(h)=(0, \ldots, h, \ldots,-h, \ldots, 0)$, where the $h,-h$ occur at the coordinates $a$ and $b$ corresponding to the fixed points $F_{a}, F_{b} \in F$ at the two ends of the orbit $E_{\tau}$. (Reversing the order of $F_{a}, F_{b}$ changes $\psi_{t}$ by a sign, but it does not affect the argument which follows.)

After translating it to homology by dualizing, Proposition 2.1(iv) says (among other things) that the following sequence is exact:

$$
\bigoplus_{\tau} \mathfrak{f}_{\tau} \stackrel{\Sigma_{\tau} \psi_{\tau}}{\longrightarrow} H_{2}^{T}(F ; \mathbb{R}) \cong \bigoplus_{j=1}^{k} \mathfrak{f} \stackrel{\sum_{j} \phi_{j}}{\longrightarrow} H_{2}^{T}(X) \longrightarrow 0 .
$$

In the first sum, $\tau$ varies over the one-dimensional orbits in $X$ or equivalently, over the codimension one cones in $\Sigma$ and the second sum is indexed by the fixed points $F_{j} \in F$. If $\bar{\tau}=\bar{C}_{a} \cap \bar{C}_{b}$ is a codimension one cone and if $h \in \mathfrak{f}_{\tau}$, then exactness of this sequence implies that $\phi_{a}(h)+\phi_{b}(-h)=0$ as claimed.

Now suppose $X$ is rationally non-singular. Then $X$ is equivariantly formal, $\widehat{H}^{*}(C)=H^{*}(X)$, and each cone $C$ in $\Sigma$ is simplicial. Moreover, the number of 
one-dimensional cones in $\Sigma$ coincides with the dimension $\beta_{2}$ of $H_{2}^{T}(X)$. In fact the Poincaré polynomial of $X$ is given [12] by

$$
P(q)=\sum_{j=1}^{2 n} \beta_{j} \sqrt{q}^{j}=\sum_{j=1}^{n} c_{n-j}(q-1)^{j},
$$

where $c_{n-j}$ denotes the number of $n-j$-dimensional cones in $\Sigma$. By Poincaré duality, $\beta_{2}=\beta_{2 n-2}=c_{1}-n$ as claimed.

On the other hand, the vectors $\Phi\left(v_{1}\right), \ldots, \Phi\left(v_{m}\right) \subset H_{2}^{T}(X ; \mathbb{R})$ cannot lie in a proper subspace, otherwise $\Phi(C)$ would also lie in this subspace, for every cone $C$ in $\Sigma$, which implies that $V_{\mathbb{R}}$ is also contained in this subspace. This contradicts Theorem 3.1 So we have shown that the vectors $\Phi\left(v_{1}\right), \ldots, \Phi\left(v_{m}\right)$ form a basis of $H_{2}^{T}(X ; \mathbb{R})$.

Let $\Sigma_{1}$ denote the collection of all the one-dimensional cones in $\Sigma$ and let $\mathcal{S}$ denote the collection of all subsets $S \subset \Sigma_{1}$ with the following property: if $S=$ $\left\{\sigma_{1}, \sigma_{2}, \ldots, \sigma_{r}\right\}$, then there exists an $n$-dimensional cone $C \in \Sigma_{n}$ such that $\bar{C}$ contains the one-dimensional cones $\sigma_{1}, \ldots, \sigma_{r}$. Because the fan $\Sigma$ is simplicial, every collection $S \in \mathcal{S}$ of one-dimensional cones spans (the closure of) a unique cone $C=\operatorname{span}(S) \in \Sigma$. The mapping span: $\mathcal{S} \rightarrow \Sigma$ is a bijection of partially ordered sets.

There is also a mapping $\Theta$ from $\mathcal{S}$ to the set of all subspaces of $H_{2}^{T}(X ; \mathbb{R})$ which associates with any collection $S=\left\{\sigma_{1}, \ldots, \sigma_{r}\right\}$ of one-dimensional cones, the subspace spanned by $\Phi\left(v_{1}\right), \ldots, \Phi\left(v_{r}\right)$. Because these vectors are linearly independent, this mapping commutes with intersection, $\Theta\left(S_{1} \cap S_{2}\right)=\Theta\left(S_{1}\right) \cap \Theta\left(S_{2}\right)$, and it is compatible with the partial ordering defined by containment. We claim that the image of $\Theta$ is precisely the partially ordered set of flats in the arrangement $V$. For if $S \in \mathcal{S}$ is such a collection of one-dimensional cones, then

$$
\operatorname{span}(S)=\bigcap\left\{C_{i} \in \Sigma_{n}: \operatorname{span}(S) \subset \bar{C}_{i}\right\},
$$

where $\Sigma_{n}$ is the collection of top-dimensional cones in $\Sigma$. (In other words, each cone is the intersection of the maximal cones containing it.) Therefore, the subspace $\Theta(S)$ is the intersection of the corresponding maximal subspaces $\mathfrak{f}_{i}$.

We remark that together with Theorem 3.1(v), this gives an alternate proof of the theorem of Brion-Vergne [9] that the equivariant cohomology $H_{T}^{*}(X ; \mathbb{R})$ of any rationally smooth toric variety is naturally isomorphic to the algebra of continuous, piecewise polynomial (real-valued) functions on the corresponding fan $\Sigma$. (For, each equivariant cohomology class corresponds to a polynomial function on the arrangement $V$, which gives a continuous piecewise polynomial function on $\Sigma$ by composing with $\Phi: \mathfrak{f} \rightarrow V \subset H_{2}^{T}(X ; \mathbb{R})$. Conversely, every continuous piecewise polynomial function on $\Sigma$ is determined by its values on the one-dimensional rays $\left.\Phi\left(L_{1}\right), \ldots, \Phi\left(L_{m}\right).\right)$

\section{Springer Fibers for $S L(n, \mathbb{C})$}

\subsection{Recollections on Springer fibers}

Let $G=S L(n, \mathbb{C})$. Let $B \subset G$ be a Borel subgroup. Each element $a \in \mathfrak{g}$ of the Lie algebra of $G$ determines a vector field $V_{a}$ on the flag manifold $X=G / B$ whose zero 
set we denote by $X_{a}$. If $a \in \mathfrak{g}$ is nilpotent, then $X_{a}$ is referred to as a "Springer fiber". T. A. Springer [30,31] constructed a surprising representation of the Weyl group $W$ of $G$ on the cohomology $H^{*}\left(X_{a}\right)$ of each Springer fiber. This representation has since been constructed by other means $[20,21]$ and it has been thoroughly analyzed. Each Springer fiber $X_{a}$ admits an action of a certain algebraic torus $T$. In this section we will show that Springer's representation has a canonical lift to a representation of $W$ on the equivariant cohomology $H_{T}^{*}\left(X_{a}\right)$. (See [16] for analogous results in the case of affine Springer fibers.)

Let us first recall some standard facts concerning the flag manifold and Springer fibers for $S L(n, \mathbb{C})$. If $H \subset B$ is a Cartan subalgebra with Lie algebra $\mathfrak{h}$, then there is a natural identification $\mathfrak{h} \cong H_{2}(X ; \mathbb{C})$ whose dual $\mathfrak{h}^{*} \cong H^{2}(X ; \mathbb{C})$ associates with each character $\chi: T \rightarrow \mathbb{C}^{\times}$the first Chern class $c^{1}\left(\mathcal{L}_{\chi}\right) \in H^{2}(X ; \mathbb{C})$ of the corresponding line bundle $\mathcal{L}_{\chi}$ on $X=G / B$.

For any nilpotent $a \in \mathfrak{g}=\mathfrak{s l}(n, \mathbb{C})$, there exists $[13,28]$ a paving of the flag manifold $X$ by affine spaces so that the Springer fiber $X_{a}$ is a closed union of affines. It follows that $H_{i}\left(X_{a}\right) \rightarrow H_{i}(X)$ is injective and $H^{i}(X) \rightarrow H^{i}\left(X_{a}\right)$ is surjective for all $i$. In particular, $\widehat{H}^{*}\left(X_{a}\right)=H^{*}\left(X_{a}\right)$ (since the same is true for $X$ ). Moreover, provided $a$ is not regular, the mapping induced by inclusion

$$
H_{2}\left(X_{a}\right) \rightarrow H_{2}(X) \cong \mathfrak{h}
$$

is an isomorphism. This is because the mapping is injective and $W$-equivariant (with respect to Springer's action on $H_{2}\left(X_{a}\right)$ ), but $W$ acts irreducibly on $\mathfrak{h}$.

Every nilpotent element $a \in \mathfrak{g}=\mathfrak{s l}(n, \mathbb{C})$ is a Richardson nilpotent. In other words, there exists a Levi subgroup $L$ of a parabolic subgroup $P \subset G$ such that $a \in \mathfrak{I}=\operatorname{Lie}(L)$ is a regular nilpotent element, i.e., $a$ lies in the largest nilpotent conjugacy class in $I$. Then the center $T$ of $L$ is a torus in $G$ which acts on the Springer fiber $X_{a}$ with finitely many fixed points. In fact (see [10]), the fixed point set $X^{T}$ (of the action of $T$ on the flag manifold $X$ ) consists of $\left[W: W_{L}\right]$ copies of the flag manifold $X_{L}$, each of which intersects the Springer fiber $X_{a}$ in a single point. (Here $W_{L}$ is the Weyl group for $L$ and, if $B$ is chosen so that $T \subset B \subset P$, then $X_{L}=L / L \cap B$.)

Now consider the arrangement of linear spaces $V \subset H_{2}^{T}(X) \rightarrow \mathrm{t}$. Using the canonical splitting (3.2), we obtain a commutative diagram,

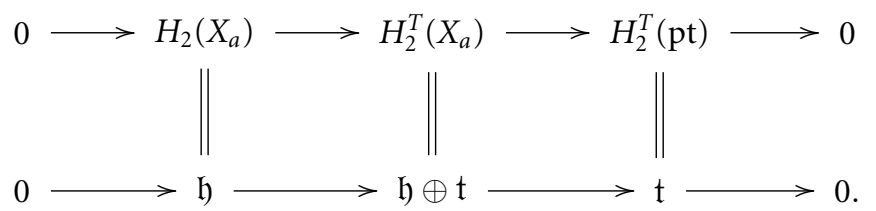

In this case, Theorem 4.1] is a theorem of Carrell [10].

Theorem 7.1 Let $a \in \mathfrak{g}$ be a non-regular nilpotent element with Springer fiber $X_{a}$. Let $T \subset L$ be the associated Richardson data. Let $t \in \mathrm{t}$ be a generic element. Then the scheme $V_{t} \subset H_{2}(X)$ is reduced and it consists of the $\left[W: W_{L}\right]$ points $W \cdot t$. The filtration 
On the Spectrum of the Equivariant Cohomology Ring

by degree on the coordinate ring $A_{t}=\mathbb{C}[W \cdot t]$ of this Weyl group orbit induces an isomorphism of rings,

$$
\mathrm{Gr} A_{t} \cong H^{*}\left(X_{a} ; \mathbb{C}\right)
$$

Proof The action of $T$ on $X_{a}$ is equivariantly formal, has isolated fixed points, and $\widehat{H}^{*}\left(X_{a}\right)=H^{*}\left(X_{a}\right)$. So by Theorem 3.1, the corresponding scheme

$$
V=\operatorname{Spec} H_{T}^{*}\left(X_{a}\right) \subset H_{T}^{2}\left(X_{a}\right)
$$

is reduced and the projection $\pi: V \rightarrow \mathrm{t}$ is flat. Therefore, Theorem 7.1 will follow immediately from Theorem 4.1, provided that we can establish the identification $V_{t}=W \cdot t$. This is most directly accomplished by first considering the case of $X=G / B$. Let us take $B$ to be the group of upper triangular matrices and $H$ to be the Cartan subgroup of diagonal matrices of determinant 1 . The Weyl group $W \cong S_{n}$ may be realized as the subgroup of permutation matrices in $G$ and also as the group of permutations of the set $\{1,2, \ldots, n\}$. It acts linearly on $\mathbb{C}^{n}$ by permuting the coordinate axes so we obtain an induced action on the flag manifold $X$. For any $\lambda \in H$ we have

$$
w \cdot(\lambda \cdot x)=\left(w \lambda w^{-1}\right) \cdot(w \cdot x)
$$

for any $x \in X$. But $w \lambda w^{-1}$ is the reflection action of $W$ on $H$. So induced action on $H_{*}(X)$ is trivial, but the induced action on $H_{*}^{H}(X)$ is non-trivial and the projection $\pi_{*}: H_{2}^{H}(X) \rightarrow H_{2}^{H}(\mathrm{pt}) \cong \mathfrak{h}$ is $W$-equivariant with respect to the reflection representation on $\mathfrak{h}$.

A second action (the Springer action) of $W$ on $X$ may be defined by first choosing a Hermitian metric on $\mathbb{C}^{n}$ that is invariant under $W$ and also under the maximal compact (connected) subtorus $K \subset H$. Then each flag

$$
0=P_{0} \subset P_{1} \subset \cdots \subset P_{n}=\mathbb{C}^{n}
$$

gives rise to an orthogonal decomposition $\mathbb{C}^{n}=M_{1} \oplus \cdots \oplus M_{n}$ such that $P_{k}=\bigoplus_{j=1}^{k} M_{k}$. Let $W$ act by permuting the $M$ 's. This determines an action from the right on $X$ by $P_{k} \cdot w=\bigoplus_{j=1}^{k} M_{w k}$ for each permutation $w$. This action is not algebraic, but it commutes with the action of $H$ so it also determines an action (from the right) on $H_{2}(X) \cong \mathfrak{h}$ which turns out to be the right-reflection representation, that is, $a \cdot w=w^{-1} a$ for $a \in \mathfrak{h}, w \in W$.

The fixed points of the action of $H$ on $X$ are the coordinate flags. If $x_{0} \in X$ denotes the standard coordinate flag $0 \subset \mathbb{C}^{1} \subset \mathbb{C}^{2} \subset \cdots \subset \mathbb{C}^{n}$ then the other fixed points are $\tau x_{0}$ for $\tau \in W$. Using the canonical splitting (3.2), we obtain a commutative diagram

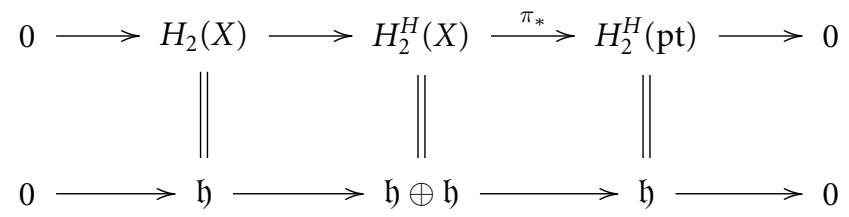


such that $W$ acts on the first factor (and on $H_{2}(X)$ ) via the right action, and $W$ acts on the second factor (and on $H_{2}^{H}(\mathrm{pt})$ via the left action. If $x_{1} \in G / B$ denotes the base point (that is, the standard flag in $\mathbb{C}^{n}$ ), then (following a calculation which we omit) the splitting corresponding to this point turns out to be

$$
(b, b) \leftarrow b
$$

The arrangement of linear spaces $V \subset H_{2}^{H}(X)$ is clearly preserved under both actions of $W$, so it follows that

$$
V=\bigcup_{\tau \in W}\{(\tau b, b): b \in \mathfrak{h}\}
$$

Consequently the fiber of $\pi_{*}$ is

$$
\pi_{*}^{-1}(t)=\{(\tau t, t): \tau \in W\} .
$$

For the Springer fiber $X_{a} \subset X$ the diagram (7.1) consists of subspaces of the diagram 7.1, so if $t \in \mathrm{t}$, then $V_{t}=\pi_{*}^{-1}(t)$ is still described by (7.3). (The only change is that the subgroup $W_{L}$ acts trivially on $t$.)

We see from the preceding proof that, in fact, we have a commutative diagram

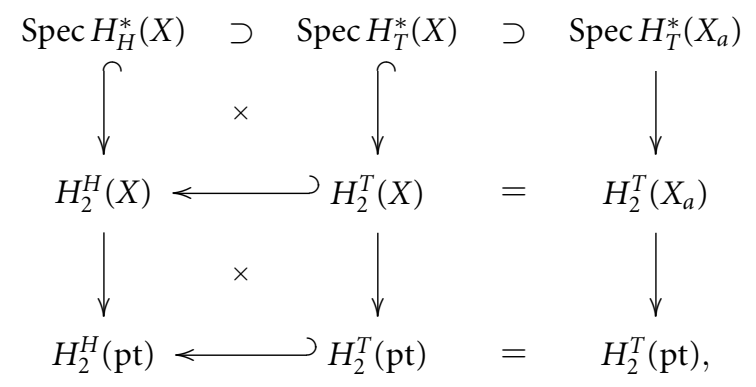

where the squares marked $\times$ are fiber squares. In other words, Spec $H_{T}^{*}(G / B)$ is the scheme-theoretic pullback of the scheme Spec $H_{H}^{*}(G / B)$ under the inclusion $\mathfrak{t} \rightarrow \mathfrak{h}$. The scheme $\operatorname{Spec} H_{T}^{*}(G / B)$ is not necessarily reduced.

Theorem 7.2 Let $X$ denote the variety of complete flags in $\mathbb{C}^{n}$. Let $a \in \mathfrak{s l}(n, \mathbb{C})$ be a non-zero nilpotent, let $T \subset L$ be the associated Richardson torus in the centralizer of $a$, and let $X_{a}$ be the associated Springer fiber. (It determines the element a up to conjugacy.) Then

$$
\operatorname{Spec}\left(H_{T}^{*}\left(X_{a}\right)\right)=\left(\operatorname{Spec} H_{T}^{*}(X)\right)_{\mathrm{red}}
$$

as sub-schemes of $H_{2}^{T}(X)$. Moreover, the Springer action on $H^{*}(X) \rightarrow H^{*}\left(X_{a}\right)$ lifts canonically to an action on the equivariant cohomology $H_{T}^{*}(X) \rightarrow H_{T}^{*}\left(X_{a}\right)$, and it determines an identification of the Weyl group,

$$
W \cong \operatorname{Aut}_{\mathbb{C}[\mathrm{t}]} H_{T}^{*}\left(X_{a}\right)
$$


On the Spectrum of the Equivariant Cohomology Ring

Proof The fixed points of the action of $T$ on $X$ determine an arrangement $V(X) \subset \mathfrak{h} \oplus \mathfrak{h}=H_{2}^{T}(X) \cong H_{2}^{T}(X)$. This coincides with the arrangement $V(X)$ because each connected component of $X^{T}$ intersects $X_{a}$ in a single fixed point. Consequently the scheme $\operatorname{Spec} H_{T}^{*}\left(X_{a}\right)$ is reduced and this proves (7.4).

The action of $W$ on $H_{2}^{T}(X)$ preserves the reduced variety $V=\operatorname{Spec} H_{T}^{*}\left(X_{a}\right)$ and the projection $\pi_{*}: H_{2}^{T}(X) \rightarrow \mathrm{t}$ is $W$-invariant. Hence this action passes to an action on $H_{T}^{*}\left(X_{a}\right)$ which is compatible with the Springer action on $H_{T}^{*}(X)$ according to the above diagram. In particular, $W \subset \operatorname{Aut}_{\pi_{*}}(V)$. Since $W$ acts transitively on the components $t_{i}$ of $V$, it also accounts for the full group of automorphisms.

\section{An Unusual Duality}

\subsection{Dual Arrangements}

If $V$ is a complex vector space let $V^{\vee}=\operatorname{Hom}(V, \mathbb{C})$ be the dual vector space. If $L_{1}, L_{2}, \ldots, L_{k} \subset V$ is a finite collection of linear subspaces, define the dual arrangement $\left(V^{\vee}, L_{1}^{\perp}, L_{2}^{\perp}, \ldots, L_{k}^{\perp}\right)$ by $L_{i}^{\perp}=\operatorname{ker}\left(V^{\vee} \rightarrow L_{i}^{\vee}\right)$.

Let us say that an arrangement $L_{1}, L_{2}, \ldots, L_{k} \subset V$ is fibered by a surjective linear map $\pi: V \rightarrow T$ if each $L_{i}$ is a section of $\pi$. Let $K=\operatorname{ker}(\pi)$. The arrangement $L_{1}, \ldots, L_{k} \subset V$ is fibered by $\pi$ if and only if $K$ is a complement to each $L_{i}$ in $V$. This holds if and only if $K^{\perp} \subset V^{\vee}$ is a complement to each $L_{i}^{\perp} \subset V^{\vee}$. Therefore, the dual arrangement $L_{1}^{\perp}, L_{2}^{\perp}, \ldots, L_{k}^{\perp} \subset V^{\vee}$ is also fibered: use the linear surjection $\pi^{*}: V^{\vee} \rightarrow T^{*}=V^{\vee} / K^{\perp}$.

An isomorphism of arrangements $\left(V, L_{1}, L_{2}, \ldots, L_{k}\right) \cong\left(V^{\prime}, L_{1}^{\prime}, L_{2}^{\prime}, \ldots, L_{k}^{\prime}\right)$ is a linear isomorphism $\phi: V \rightarrow V^{\prime}$ which takes $L_{i}$ isomorphically to $L_{i}^{\prime}$ (possibly after a permutation of the indices). If these arrangements are fibered by linear surjections $\pi: V \rightarrow T$ and $\pi^{\prime}: V^{\prime} \rightarrow T^{\prime}$, then the isomorphism is fibration preserving if it also takes $K=\operatorname{ker}(\pi)$ isomorphically to $K^{\prime}=\operatorname{ker}\left(\pi^{\prime}\right)$.

\subsection{Partial Flag Manifold}

As in Section 7.1, let $H \subset B \subset G=S L(n, \mathbb{C})$ be the standard Borel pair of diagonal and upper triangular matrices of determinant 1 . Let $n=n_{1}+n_{2}+\cdots+n_{k}$ be an ordered partition. It corresponds to a partial flag $E_{1} \subset E_{2} \subset \cdots \subset \mathbb{C}^{n}$ of dimensions $n_{1}, n_{1}+n_{2}, \ldots, n$. Let $P \subset G$ be the parabolic subgroup of $G$ that fixes this flag and let $L \subset G$ be its Levi subgroup consisting of block-diagonal matrices with block sizes $n_{1}, n_{2}, \ldots, n_{k}$. Let $T=H \cap L$ be its center. Let $K=S O(n, \mathbb{C})$ be the (standard) maximal compact subgroup. The diffeomorphism $G / P \cong(K \cap L) /(K \cap T)$ induces an isomorphism $H_{2}(G / P) \cong \mathrm{t}$, so we obtain an exact sequence

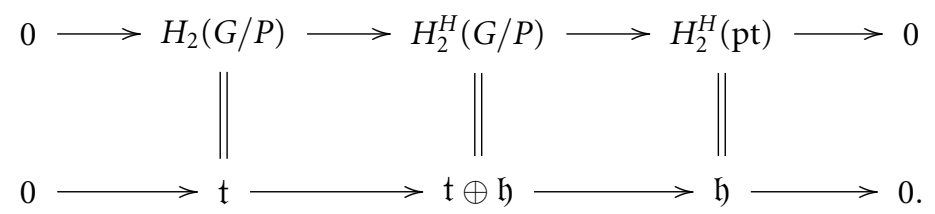


The projection $q: G / B \rightarrow G / P$ is $H$ equivariant. The induced mapping

$$
q_{*}: H_{2}(G / B) \rightarrow H_{2}(G / P)
$$

is given by orthogonal projection $\mathfrak{h} \rightarrow \mathfrak{t}$ with respect to the Killing form. The resulting mapping,

$$
H_{2}^{T}(G / B) \cong \mathfrak{h} \oplus \mathfrak{h} \rightarrow H_{2}^{T}(B / P) \cong \mathfrak{t} \oplus \mathfrak{h}
$$

takes the arrangement $V(G / B)$ to the arrangement $V(G / P)$ which can therefore be described as follows,

$$
V(G / P)=\bigcup_{\tau \in W}\left\{\left(q_{*}(\tau b), b\right) \in \mathfrak{t} \oplus \mathfrak{h}: b \in \mathfrak{h}\right\} .
$$

The fixed point set $(G / P)^{H}$ is the image under $q$ of the fixed point set $(G / B)^{H}$, so the fixed points are isolated. Hence (Theorem 3.1) the scheme $W(G / P)=$ Spec $\widehat{H}_{H}^{*}(G / P)$ is reduced. (However the projection $V(G / P) \rightarrow \mathfrak{h}$ is not flat, because $H^{*}(G / P) \neq \widehat{H}^{*}(G / P)$.)

Theorem 8.1 Let $a \in \mathfrak{s l}(n, \mathbb{C})$ be the standard nilpotent element with Jordan blocks of sizes $n_{1}, n_{2}, \ldots, n_{k}$. Let $X_{a}$ be the corresponding Springer fiber. Then the fibered arrangement

$$
\operatorname{Spec} H_{T}^{*}\left(X_{a}\right)=V\left(X_{a}\right) \subset \mathfrak{t} \oplus \mathfrak{h} \rightarrow \mathfrak{t}
$$

is isomorphic to the dual of the fibered arrangement

$$
\operatorname{Spec} \widehat{H}_{H}^{*}(G / P)=V(G / P) \subset \mathfrak{h} \oplus \mathfrak{t} \rightarrow \mathfrak{h} \text {. }
$$

Proof Let $E_{1}=\mathfrak{t} \oplus \mathfrak{h}$ and $E_{2}=\mathfrak{h} \oplus \mathfrak{t}$. There is a dual pairing $E_{1} \times E_{2} \rightarrow \mathbb{C}$ given by

$$
\left\langle\left(t_{1}, h_{1}\right),\left(h_{2}, t_{2}\right)\right\rangle_{E}=\left\langle t_{1}, t_{2}\right\rangle-\left\langle h_{1}, h_{2}\right\rangle
$$

where $\langle\cdot, \cdot\rangle$ denotes the Killing form on $\mathfrak{h}$ (and its restriction to $\mathfrak{t}$ ). With respect to this pairing, the dual to $\mathfrak{t} \oplus 0 \subset E_{1}$ is $\mathfrak{h} \oplus 0 \subset E_{2}$, and the dual to $0 \oplus \mathfrak{h} \subset E_{1}$ is $0 \oplus \mathrm{t} \subset E_{2}$. We claim that the dual to $V\left(X_{a}\right) \subset E_{1}$ is $V(G / B) \subset E_{2}$. The two arrangements contain the same number of subspaces and they have complementary dimensions. We must show that each subspace in the first arrangement is the dual of a particular subspace in the second arrangement. From equations (7.2) and (8.1) it suffices to show that for any $w \in W$, the dual of the subspace

$$
L=\left\{\left(q_{*}(w b), b\right): b \in \mathfrak{h}\right\} \subset E_{1}
$$

is the subspace $L^{\prime}=\left\{\left(w^{-1} t, t\right): t \in \mathrm{t}\right\} \subset E_{2}$. Since $q_{*}$ is the orthogonal projection to $\mathrm{t}$, for any $t \in \mathrm{t}$ and any $x \in \mathfrak{h}$ we have $\left\langle q_{*}(x), t\right\rangle=\langle x, t\rangle$. Therefore a point $(h, t) \in E_{2}$ is in $\left\{\left(q_{*}(w b), b\right): b \in \mathfrak{h}\right\}^{\perp}$ if and only if

$$
0=\left\langle\left(q_{*}(w b), b\right),(h, t)\right\rangle_{E}=\left\langle q_{*}(w b), t\right\rangle-\langle b, h\rangle=\langle w b, t\rangle-\langle b, h\rangle
$$

for all $b \in \mathfrak{h}$. Since the action of $W$ preserves the Killing form, this is equivalent to $\left\langle b, w^{-1} t\right\rangle=\langle b, h\rangle$ or $h=w^{-1} t$, which is the subspace $L^{\prime} \subset E_{2}$. 
On the Spectrum of the Equivariant Cohomology Ring

\section{References}

[1] E. Akyildiz, J. Carrell, and D. Lieberman, Zeros of holomorphic vector fields on singular spaces and intersection rings of Schubert varieties. Compositio Math. 57(1986), no. 2, 237-248.

[2] C. Allday and V. Puppe, Cohomological Methods in Transformation Groups. Cambridge Studies in Advanced Mathematics 32. Cambridge University Press, Cambridge, 1993.

[3] M. Atiyah and R. Bott, The moment map and equivariant cohomology, Topology 23(1984), no. 1, 1-28. doi:10.1016/0040-9383(84)90021-1

[4] M. Audin, The Topology of Torus Actions on Symplectic Manifolds, Progress in Mathematics 93, Birkhäuser Boston, Boston, MA, 1991.

[5] T. Braden, T. Licata, C. Phan, N. Proudfoot, and B. Webster, Goresky-MacPherson duality and deformations of Koszul algebras. arXiv:0905.1335v1.

[6] M. Brion, Equivariant cohomology and equivariant intersection theory, Notes de cours, Montréal, 1997. http://www-fourier.ujf-grenoble.fr/ mbrion/notes.html.

[7] Poincaré duality and equivariant (co)homology. Michigan Math. J. 48(2000), 77-92. doi: $10.1307 / \mathrm{mmj} / 1030132709$

[8] M. Brion and J. Carrell, The equivariant cohomology ring of regular varieties. Michigan Math. J. 52(2004), no. 1, 189-203. doi:10.1307/mmj/1080837743

[9] M. Brion and M. Vergne, An equivariant Riemann-Roch theorem for complete, simplicial toric varieties. J. Reine Angew. Math. 482(1997), 67-92.

[10] J. Carrell, Orbits of the Weyl group and a theorem of DeConcini and Procesi. Compositio Math. 60(1986), no. 1, 45-52.

[11] J. Carrell, K. Kaveh, and V. Puppe, Vector fields, torus actions and equivariant cohomology. Pacific J. Math. 232(2007), no. 1, 61-76.

[12] V. I. Danilov, The geometry of toric varieties. Russian Math. Surveys 33(1978), no. 2, 97-154.

[13] C. De Concini, G. Lusztig, and C. Procesi, Homology of the zero-set of a nilpotent vector field on a flag manifold. J. Amer. Math. Soc. 1(1988), no. 1, 15-34. doi:10.2307/1990965

[14] C. De Concini and C. Procesi, Symmetric functions, conjugacy classes, and the flag variety. Invent. Math. 64(1981), no. 2, 203-219. doi:10.1007/BF01389168

[15] W. Fulton, Introduction to Toric Varieties. Annals of Mathematics Studies 131. Princeton University Press, Princeton NJ, 1993.

[16] M. Goresky, R. Kottwitz, and R. MacPherson, Equivariant cohomology, Koszul duality, and the localization theorem. Invent. Math. 131(1998), no. 1, 25-83. doi:10.1007/s002220050197

[17] Homology of affine Springer fibers in the unramified case. Duke Math. J. 121(2004), no. 3, 509-561 doi:10.1215/S0012-7094-04-12135-9

[18] W. Hsiang, Cohomology Theory of Topological Transformation Groups. Ergebnisse der Mathematik und ihrer Grenzgebiete 85. Springer Verlag, New York, 1975.

[19] K. Kaveh, Note on the cohomology ring of spherical varieties and volume polynomial. arXiv:math/0312503.

[20] D. Kazhdan and G. Lusztig, A topological approach to Springer's representations. Adv. in Math. 38(1980), no. 2, 222-228. doi:10.1016/0001-8708(80)90005-5

[21] G. Lusztig, Green polynomials and singularities of unipotent classes. Adv. Math. 42(1981), no. 2, 169-178. doi:10.1016/0001-8708(81)90038-4

[22] A. Pukhlikov and A. Khovanskii, The Riemann-Roch theorem for integrals of sums of quasipolynomials on virtual polytopes. Algebra i Analiz 4(1992), no. 4, 188-216 (Russian); St. Petersburg Math. J. 4(1993), no. 4, 789-812 (English translation).

[23] V. Puppe, On a conjecture of Bredon. Manuscripta Math. 12(1974), 11-16. doi:10.1007/BF01166231

[24] Cohomology of fixed point sets and deformation of algebras. Manuscripta Math. 23(1977/78), no. 4, 343-354. doi:10.1007/BF01167693

[25] Deformations of algebras and cohomology of fixed point sets. Manuscripta Math. 30(1979/80), no. 2, 119-136. doi:10.1007/BF01300965

[26] — Do manifolds have little symmetry? J. Fixed Point Theory Appl. 2(2007), no. 1, 85-96. doi:10.1007/s11784-007-0021-x

[27] D. Quillen, The spectrum of an equivariant cohomology ring. I. Ann. of Math. 94(1971), 549-572. doi: $10.2307 / 1970770$

[28] N. Spaltenstein, The fixed point set of a unipotent transformation on the flag manifold. Nederl. Akad. Wetensch. Proc. Ser. A 38(1976), no. 5, 452-456. 
[29] E. Spanier, Algebraic Topology. McGraw-Hill, New York, 1966.

[30] T. A. Springer, Trigonometric sums, Green functions of finite groups and representations of Weyl groups. Invent. Math. 36(1976), 173-207. doi:10.1007/BF01390009

[31] A purity result for fixed point varieties in flag manifolds. J. Fac. Sci. Univ. Tokyo Sect. IA Math. 31(1984), no. 2, 271-282.

[32] C. Stroppel, Parabolic category O, perverse sheaves on Grassmannians, Springer Fibres and Khovanov homology. Compositio Math. 145(2009), no. 4, 954-992.

School of Mathematics, Institute for Advanced Study, Princeton, NJ, USA

e-mail: goresky@ias.edu

rdm@math.ias.edu 